\title{
Paper Mitigation of Scintillation Effects in WDM FSO System using Multibeam Technique
}

\author{
Marvi Grover, Preeti Singh, and Pardeep Kaur \\ Department of Electronics and Communication Engineering, University Institute of Engineering and Technology, \\ Panjab University, Chandigarh, India
}

\begin{abstract}
Free Space Optical communication (FSO) has engrossed a large section of researchers in recent times due to its wide bandwidth, effortless deployment and immune links making it appropriate for communication purposes. This wireless optical technique requires clear and non-turbulent atmospheric conditions for efficient transmission. In this paper, authors aim at reducing the effect of turbulent atmospheric conditions like scintillation effect on FSO. Multibeam technique, which uses spatially diverse transmitters for transmission, has been used for increasing the achievable link distance of the FSO system. Parameters like quality factor and bit error rate have been used to check the received signal quality.
\end{abstract}

Keywords—laser, link length, multibeam, scintillation.

\section{Introduction}

Free Space Optical communication (FSO) or sometimes addressed as laser communication is a technology that uses laser beams through free space to reach the receiver. This technology owes its growing importance to the incredible increase in the volume of data transfer all over the world and the resultant increase in bandwidth requirements. FSO's key attributes like rapid data transfer, quicker deployment, cost effective infrastructure and data rates as high as tens of gigabytes per 1 second make it a viable alternative for the short-range radio frequency (RF) links [1], [2]. Licensed frequency bands, spectrum congestion and lesser data rates as compared to FSO, are some of the demerits of RF. Nowadays, FSO is finding its application in almost every stratum of daily life, ranging from ship to ship communication to enterprise connectivity [3].

Like every other technology FSO also has some limitations and some design considerations which need to be contemplated. Light beam carrying the information travels through air and is encumbered by the atmospheric effects, like rain, fog, snow, haze, and the atmospheric turbulences due to temperature and pressure fluctuations in the atmosphere [4]. Absorption, scattering and scintillation of light are consequences of turbulent atmospheric conditions [5]. Line of sight (LOS) is an imperative requirement in FSO communication, but sometimes physical objects like birds or poles temporarily obstruct it, making the link unachievable.
This paper focuses on the impairments caused by atmospheric effects on an FSO link. When considering the atmospheric effects, scintillation effect is the most detrimental one, so the authors here have tried to reduce this effect using some techniques described in this paper.

A brief description of the harm caused by scintillation on the light beam is given below.

\subsection{Scintillation Effect}

Scintillation refers to the turbulence caused by thermal inhomogeneities along the path of light beam. Wind velocity is always variable, which transfers heat and water vapors in the form of eddies. Temperature changes in the atmosphere caused by these eddies lead to heating up of air pockets called Fresnel zones having different temperatures and different densities, which lead to refractive index differences [5]. Turbulences are random, which means that these pockets are continuously being created and destroyed. Fluctuations in the refractive index of air deform the laser beam causing "beam dancing" at the receiver. Figure 1 shows the scintillation effect with air pockets having different refractive indices. Randomly formed pockets refract the optical wavefront of the incoming beam due to which the signal cannot be received properly [6]. The refractive index structure parameter $C_{n}^{2}$, accounts for the strength of fluctuations. $C_{n}^{2}$ varies from $10^{-16} \mathrm{~m}^{-2 / 3}$ (weak scintillation) to $10^{-12} \mathrm{~m}^{-2 / 3}$ (strong).

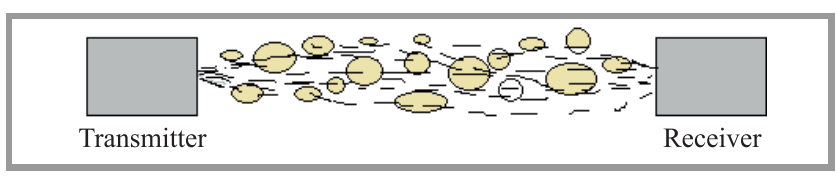

Fig. 1. Heated air pockets which lead to scintillation of light.

Two common effects of scintillation on the optical beam are:

- Beam Wander - the refractive index fluctuations are due to turbulent eddies of size varying from few millimeters to hundred meters. Beam wander means that the beam is deflected from its original path and loses its los. It happens when the size of refractive index inhomogeneity is greater than the beam diameter; 
- Beam Spreading - when the inhomogeneities are lower than the size of beam diameter, they tend to broaden the beam but do not deflect it. This is called beam spreading. It defocuses the beam reducing its intensity.

In communication systems, bandwidth is always a factor that needs deliberation, so only the mitigation of channel turbulence like scintillation effect does not solve the purpose. It should be combined with efficient bandwidth utilization in order to make it a quintessential system. One of the best techniques used here is Wavelength Division Multiplexing (WDM).

\section{WDM Systems}

WDM allows multiplying data streams over optical carriers having different wavelengths called channels and sent as a single signal. WDM FSO systems use a single light beam to transmit the multiplexed signal through free space [7]. A multiplexer is used at the transmitter to combine different modulated carriers and a demultiplexer at the receiver to restore each one (Fig. 2).

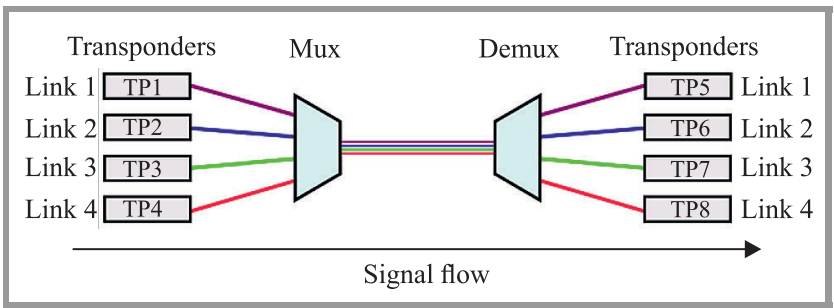

Fig. 2. WDM technology.

WDM system used in combination with FSO are called WDM FSO and can be classified into two types: single beam and multibeam systems.

Single beam system uses one pair of transmitter and receiver. Only one beam carrying the information travels through the channel. In case of FSO systems, if the light beam is obstructed by an object, which prevents it from reaching the receiver, the signal is lost and communication stops.

The multibeam WDM uses more than one beams of the multiplexed signal. Each beam travels a different path, and thus its attenuation is different. This technique uses spatially diverse transmitters and so it is also called Spatial Diversity Technique [8], [9]. At the receiver, the beam that has undergone least attenuation is selected and processed for data extraction. This technique serves as a solution for various FSO limitations like physical obstructions, scintillation effect, weather effects, etc. Multibeam system improves the link achievability and reduces the probability of link failure to a large extent [10]-[12]. When WDM FSO system uses multiple beams for transmission, they are called "Hybrid multibeam WDM FSO systems". Figure 3 shows a hybrid multibeam WDM FSO, which combines the

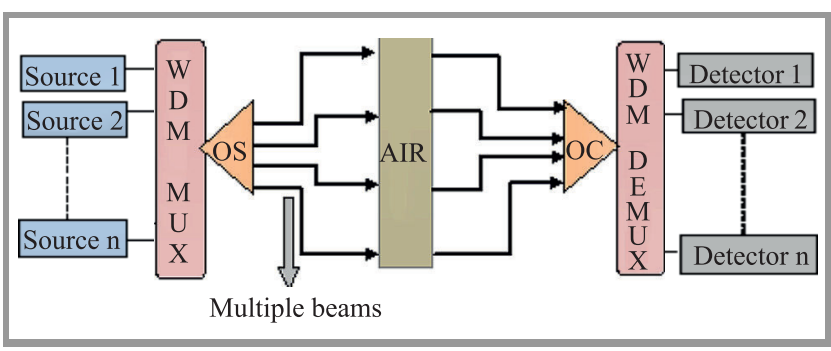

Fig. 3. Multibeam WDM FSO system block diagram.

advantages of WDM and spatial diversity to increase the system capacity and link reliability.

\section{System Design and Analysis}

In this paper two WDM FSO systems are used and analyzed under scintillation effect. First is WDM FSO, which uses single beam technology and system 2 uses the multibeam technology. System 1 has been already used by the authors in [14]. System 2 has been designed with an aim to improve the efficiency of system 1 under identical atmospheric conditions. Quality factor (Q) and bit error rate (BER) have been used as the measures of received signal quality. Comparative analysis of both systems has been done in terms of link distance and received power for best values of quality factor and BER. The software used for analysis are OptiSystem v12 and Matlab.

\subsection{System Model}

Figure 4 shows the layout of system 1 designed in OptiSystem software. The transmitter section consists of continuous wave $(\mathrm{CW})$ laser source. The fork component is used to copy the signal generated by the laser source so that it can be given to the multiplexer, which separates it into carriers differing in wavelength. The pseudo-random bit sequence (PRBS) source is used to generate codes corresponding to the information signal. It is followed by non-return to zero (NRZ) pulse generator, which gives the electrical pulses for the signal generated by the PRBS using NRZ pulse generation format. The Mach-Zehnder modulator (MZM) does the modulation and next the modulated signal is transmitted through the free space channel. At the receiver, a demultiplexer is installed with signal carrier selects then the photodetector for conversion to electrical signal. In next block the signal is filtered, regenerated and sent to the corresponding user. BER analyzer is used to view the quality factor, BER value calculation and eye diagram of the received signal.

System 2 differs from system 1 only in the way of transmission after modulation. As this is a multibeam system, it uses spatially diverse transmit apertures to transmit the signal. As shown in the Fig. 5, a fork is used after MZM modulator to simulate four different transmit apertures and a single receiver lens, which make it a $4 \times 1$ WDM FSO system. The four beams transmitted are identical but travel 


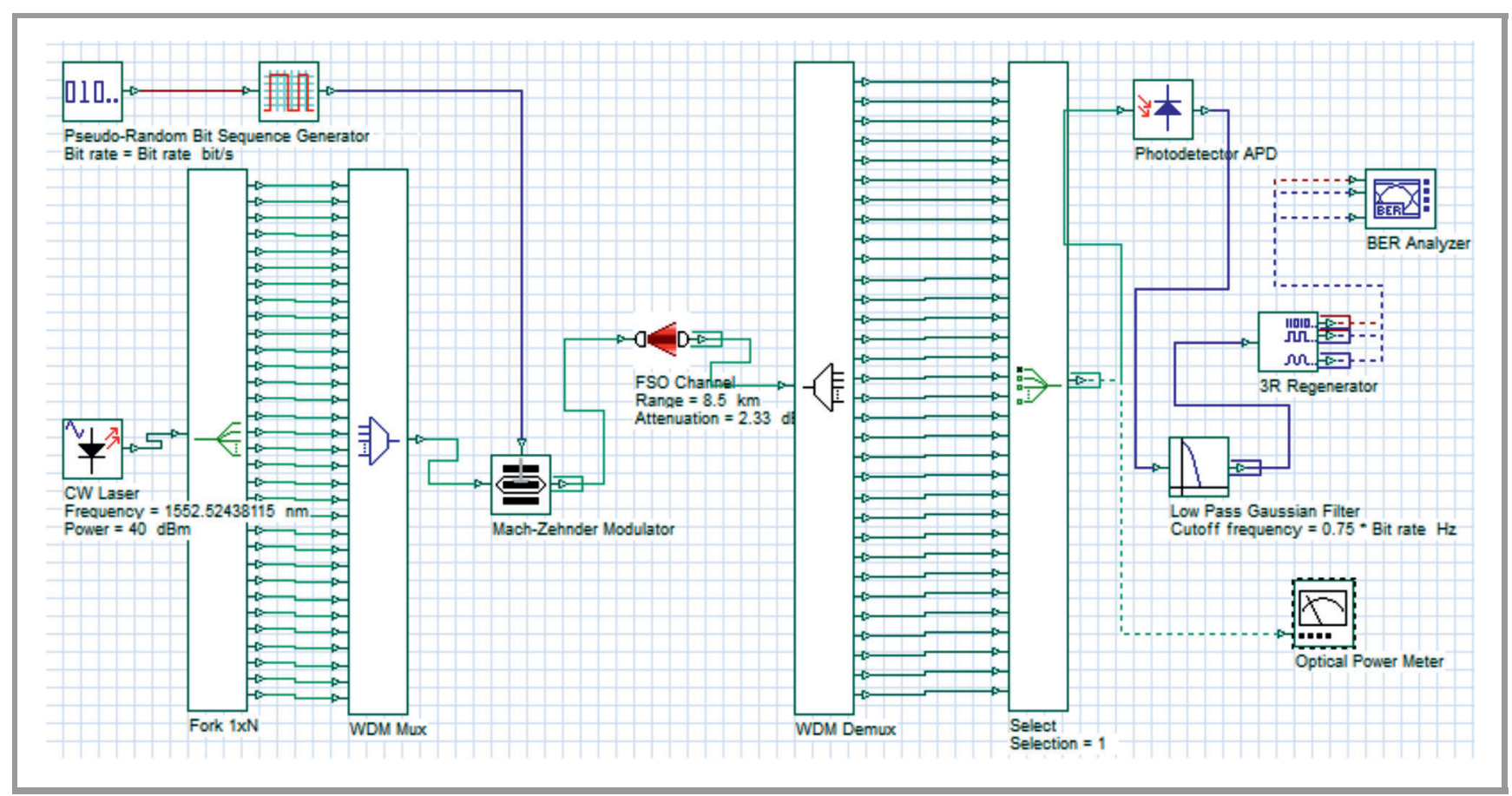

Fig. 4. System 1 designed in OptiSystem.

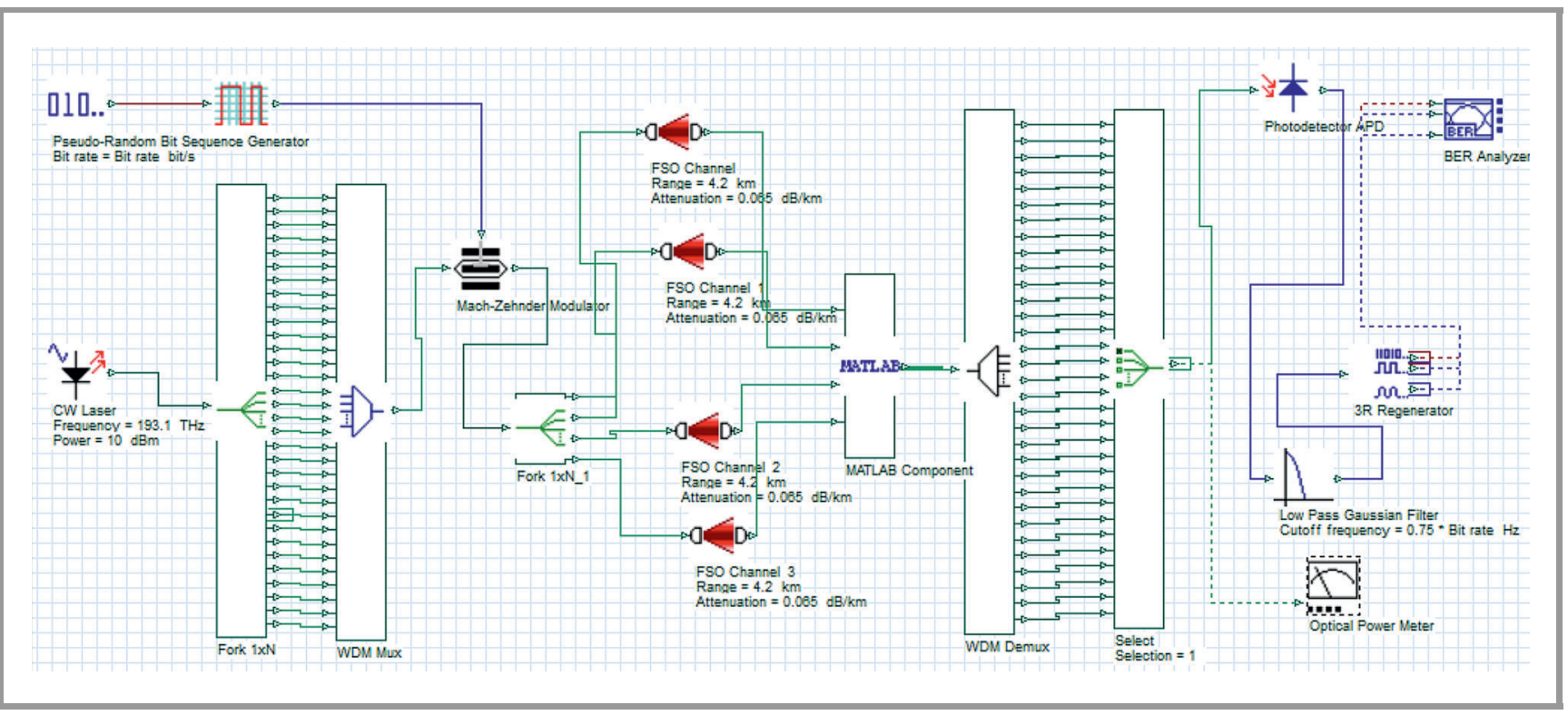

Fig. 5. System 2 schematic.

different paths to the receiver and thus undergo different amount of scintillation. Figure 5 shows a MATLAB component, which intakes the incoming signals at the receiver, selects the least attenuated out of the four, and sends it to the demultiplexer.

The CW laser operates at $1550 \mathrm{~nm}$, used data rate is $10 \mathrm{~Gb} / \mathrm{s}$, transmitter and receiver lens apertures are kept as $15 \mathrm{~cm}$. Geometrical loss has also been considered in the analysis, so the beam divergence is taken to be $2 \mathrm{mrad}$. There are various models available, for mathematical modeling of the turbulence affected FSO channel. These models give the probability density function (PDF) of the re- ceived signal after passing through the turbulent atmospheric conditions. When the channel is affected by weak turbulence it is modeled using log-normal model. In case of strong turbulence in the channel negative exponential model and K-turbulence model are used [13]. This work has been done using "gamma-gamma" turbulence model [14], which is used when the turbulence varies from moderate to strong.

The gamma-gamma model is used to model the irradiance of optical channels for moderate to strong turbulence channels resulting from small scale and large scale refractive index fluctuations due to temperature and pres- 
sure inhomogeneities. The PDF of the turbulent channel is given by:

$$
P(I)=\frac{2(\alpha \beta)^{\frac{\alpha+\beta}{2}}}{\Gamma(\alpha) \Gamma(\beta)} I^{\frac{\alpha+\beta}{2}-I} K_{\alpha-\beta}(2 \sqrt{\alpha \beta I}),
$$

where $\frac{1}{\alpha}$ and $\frac{1}{\alpha}$ are the variances of the small scale and large scale eddies respectively, $\Gamma$ is the gamma function and $K_{\alpha-\beta}(\ldots)$ is the modified second order Bessel function. $I$ is the intensity of the received signal.

Equations (2)-(3) give the values of $\alpha$ and $\beta$ respectively:

$$
\begin{gathered}
\alpha=\mathrm{e}^{\frac{0.49 \sigma_{r}^{2}}{\left(1+1.11 \sigma_{r}^{\frac{12}{5}}\right)^{\frac{5}{6}}}-1,} \\
\beta=\mathrm{e}^{\frac{0.51 \sigma_{r}^{2}}{\left(1+0.69 \sigma_{r}^{\frac{12}{5}}\right)^{\frac{5}{6}}}}-1,
\end{gathered}
$$

where $\sigma_{r}^{2}$ is the Rytov variance, which characterizes the strength of turbulence and is calculated by:

$$
\sigma_{r}^{2}=1.23 C_{n}^{2} k^{\frac{7}{6}} z^{\frac{11}{6}}
$$

where $k$ is the wave number, $z$ is the range of the link, and $C_{n}^{2}$ is the refractive index structure parameter, which is the qualitative measure of optical turbulence.

\section{Performance Analysis}

With the effect of scintillation depends on the refractive index structure parameter $C_{n}^{2}$, which is given as a parameter to the FSO channel and the signal is attenuated according to the value of $C_{n}^{2}$. For system $1, C_{n}^{2}$ is taken to $10^{-13} \mathrm{~m}^{-\frac{2}{3}}$, which corresponds to strong turbulence. When simulated for refractive index structure parameter, the maximum link distance achieved with acceptable quality factor is $1.9 \mathrm{~km}$. The Q factor value for this distance was recorded to be 5.96 and the BER was $1.21 \cdot 10^{-9}$. Weather is assumed to be clear to see the effect of scintillation, so in the attenuation specification of the FSO channel, the value given is 0.065 $\mathrm{dB} / \mathrm{km}$.

Multibeam WDM FSO system uses four beams of the system propagate independently hence, suffer different amount of scintillation, which depends upon the refractive index structure parameter. The value of $C_{n}^{2}$ used for the four beams is $10^{-16}, 10^{-15}, 10^{-14}, 10^{-13} \mathrm{~m}^{-\frac{2}{3}}$ to represent that the beams undergo different scintillation eddies due to their different propagation paths. This system works efficiently up to $4.2 \mathrm{~km}$ with the Q factor of 5.94 and BER of $1.44 \cdot 10^{-9}$. If the distance is further increased, the $\mathrm{Q}$ factor falls below its value for successful communication.

\section{Results Discussion}

Both systems have been compared in terms of $\mathrm{Q}$ factor value and received optical power. Figures 6 and 7 present systems performance in terms of $\mathrm{Q}$ factor variation with respect to link distance and illustrates the difference in quality of received signal at various link lengths. Graph shows that system 1 works till around $1.9 \mathrm{~km}$ whereas for system 2, signal quality is acceptable up to $4.2 \mathrm{~km}$.

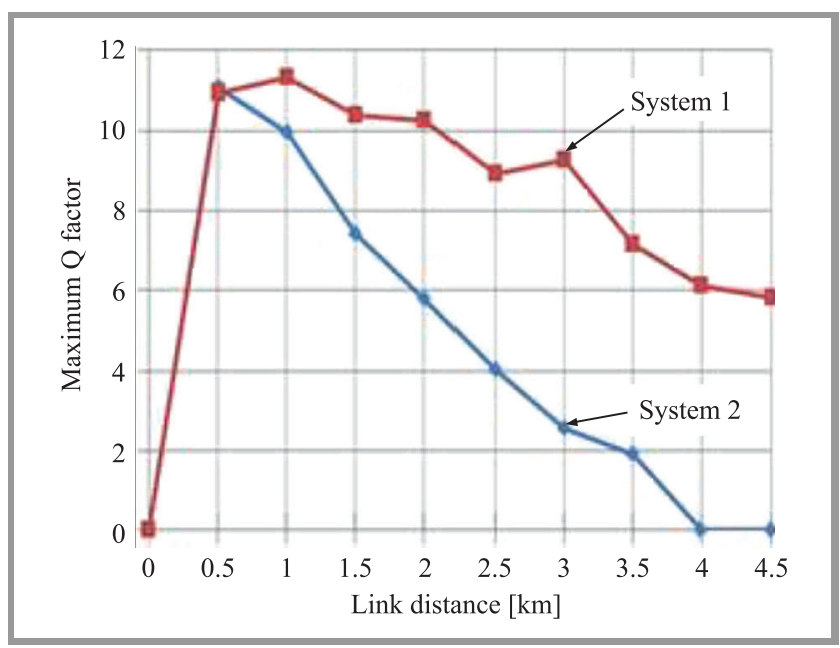

Fig. 6. Comparison of system 1 and 2 under scintillation effect in terms of maximum $\mathrm{Q}$ factor.

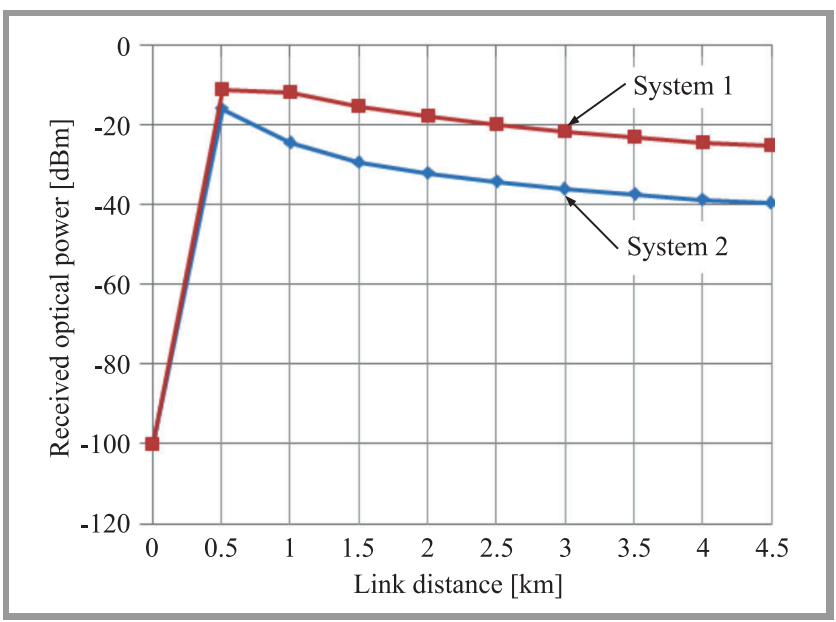

Fig. 7. Comparison of system 1 and 2 under scintillation effect in terms of received optical power.

Graph comparing the received power for both the systems (Fig. 7) shows that the received optical power of system 2 is always greater than that of system 1 when plotted against the link distance. Both the graphs clearly favor the performance of system 2, when analyzed under scintillation effect. Both systems have also been compared using the eye diagrams. Figure 8 shows the eye diagrams of both the systems at $1.9 \mathrm{~km}$ and show that the $\mathrm{Q}$ factor of system 2 (the red curve in the diagram) is much higher than that of system 1, also the eye height for system 2 is 110 , whereas that for system 1 is around 20. This difference in the eye heights also indicates better signal reception of system 2 . 

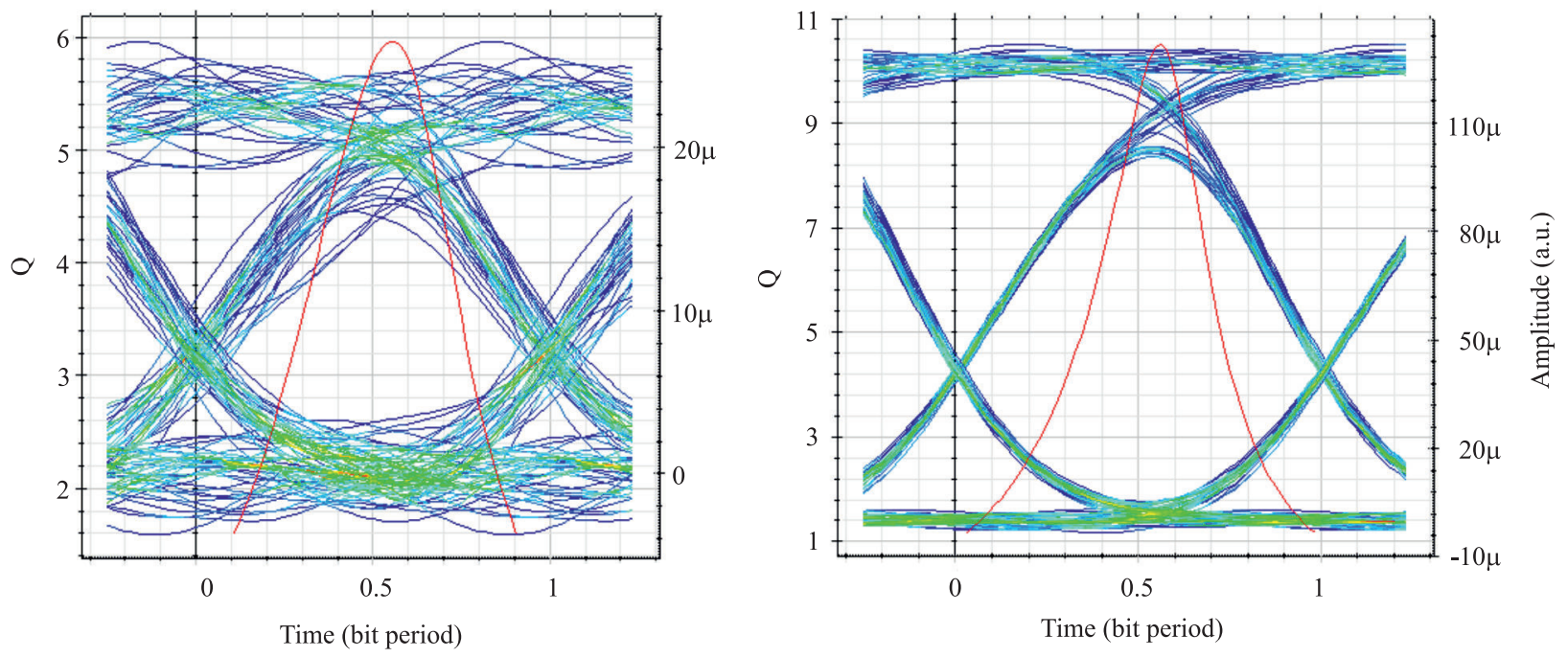

Fig. 8. Eye diagrams for system 1 (left) and 2 (right) at $1.9 \mathrm{~km}$ under scintillation effect.

Table 1

Comparison of system 1 and 2 under scintillation effect

\begin{tabular}{|c|c|c|c|c|}
\hline & $C_{n}^{2}\left[\mathrm{~m}^{-\frac{2}{3}}\right]$ & Max. link distance $[\mathrm{km}]$ & Q factor & Min. BER \\
\hline \hline System 1 & $10^{-13}$ & 1.9 & 5.94 & $1.2 \cdot 10^{-9}$ \\
\hline System 2 & $10^{-16}, 10^{-15}, 10^{-14}, 10^{-13}$ & 4.2 & 5.95 & $1.34 \cdot 10^{-9}$ \\
\hline
\end{tabular}

Both system performances and difference in the FSO link distance in Table 1 is summarized.

\subsection{Validation of Results Using Matlab}

To check the credibility of the above results, multibeam FSO links have been simulated using Matlab. The PDF of received power has been plotted against the received

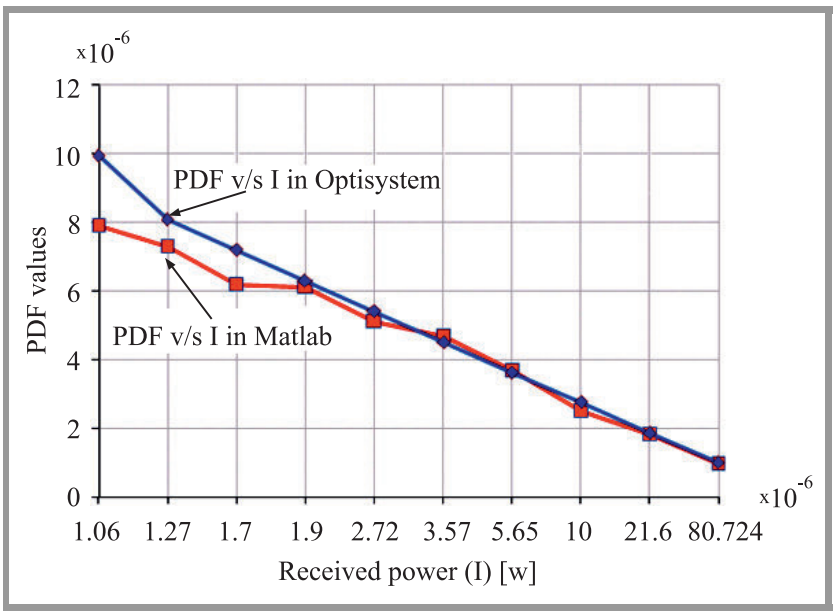

Fig. 9. Comparison of PDF vs. I curves obtained by using OptiSystem and Matlab under identical FSO channels. power $I$ using MATLAB as well as OptiSystem. Figure 9 shows the comparison graph obtained by using PDF and $I$ values both software tools.

There is a big similarity between results given by both software. Thus, it can be inferred, that the analysis done is a valid one.

\section{Conclusion}

Analysis shows that when simulated under scintillation effect, multibeam system transmits successfully up to $4.2 \mathrm{~km}$. It is much greater than that achieved by the single beam system, which transmits only up to $1.9 \mathrm{~km}$, under same atmospheric conditions. Multibeam system outperforms single beam system taking into account scintillation effect. Thus it can be used in the FSO applications where the signal reliability is important.

\section{References}

[1] D. Kedar and S. Arnon, "Urban optical wireless communication networks: The main challenges and possible solutions", IEEE Commun. Mag., vol. 42, no. 5, pp. S2-S7, 2004 (doi: 10.1109/MCOM.2004.1299334).

[2] J. Kaufmann, "Free space optical communications: An overview of applications and technologies", in Boston IEEE Communications Society Meeting CommSoc 2011, Boston, MA, USA, 2011. 
[3] A. Malik and P. Singh, "Free space optics: Current applications and future challenges", Int. J. of Optics, vol. 2015, article ID 945483 (doi: 10.1155/2015/945483).

[4] V. Sharma and G. Kaur, "Degradation measures in free space optical communication (FSO) and its mitigation techniques - A review", Int. J. of Computer Appl., vol. 55, no. 1, pp. 23-27, 2012.

[5] M. Abtahi, P. Lemieux, W. Mathlouthi, and L. A. Rusch, "Suppression of turbulence-induced scintillation in free space optical communication systems using saturated optical amplifiers", Lightwave Technol., vol. 24, no. 12, pp. 4966-4973, 2006.

[6] M. Ali and A. Ali, "Atmospheric turbulence effect on free space optical communication", Int. J. of Emerging Technol. in Comput. and Appl. Sci., vol. 5, no. 4, pp. 345-351, 2013.

[7] A. Malik and P. Singh, "Comparative analysis of point to point FSO system under clear and haze weather conditions", Wirel. Personal Commun., vol. 80, no. 2, pp. 483-492, 2014.

[8] A. B. Mohammad, "Optimization of FSO system in tropical weather using multiple beams", in Proc. 5th Int. Conf. on Photonics ICP 2014, Kuala Lumpur, Malaysia, 2014 (doi: 10.1109/ICP.2014.7002326).

[9] Y. Zhao, D. Xu, and X. Zhong, "Scintillation reduction using multibeam propagating technique in atmospheric WOCDMA system", Chinese Optics Lett., vol. 4, no. 11, pp. 110602-110605, 2011 (doi: 10.3788/COL201109.110602).

[10] T. A. Tsiftsis et al., "Optical wireless links with spatial diversity over strong atmospheric turbulence channels", IEEE Trans. on Wirel. Commun., vol. 8, no. 2, pp. 951-957, 2009.

[11] S. A. Al-Gailani, A. B. Mohammad, and R. Q. Shaddad, "Enhancement of free space optical link in heavy rain attenuation using multiple beam concept", Optik, vol. 124, no. 21, pp. 4798-4801, 2013.

[12] N. H. M. Noor, W. Al Khateeb, and A. W. Naji, "Experimental evaluation of multiple transmitters/receivers on free space optics link", in Proc. IEEE Student Conf. on Res. and Develop. SCOReD 2011, Cyberjaya, Malaysia, 2011 (doi: 10.1109/SCOReD.2011.6148721).

[13] X. Zhu and J. M. Kahn, "Free-space optical communication through atmospheric turbulence channels", IEEE Trans. on Commun., vol. 50, no. 8, pp. 1293-1300, 2002.

[14] D. Shah, B. Nayak, and D. Jethawani, "Study of different Atmospheric channel models", Int. J. of Electron. and Commun. Engin. \& Technol., vol. 5, no. 1, pp. 105-112, 2014.

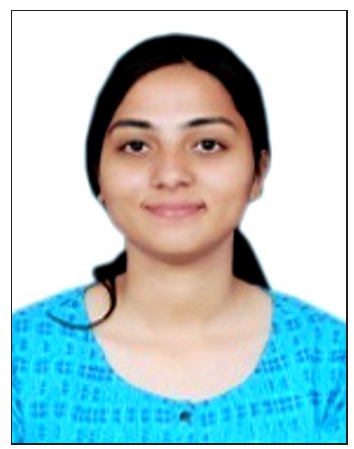

Marvi Grover has completed her M.Sc. in Electronics and Communication Engineering from U.I.E.T., Panjab University, Chandigarh, India. She has done her research in wireless optical communication. Her area of interest is free space optical communication.
E-mail: marvi310191@gmail.com

Department of Electronics and Communication

Engineering

University Institute of Engineering and Technology

Panjab University

Chandigarh, India

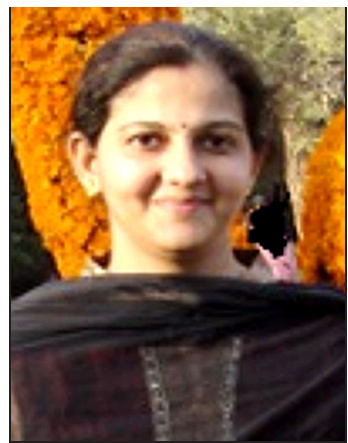

Preeti Singh is an Assistant Professor in Electronics and Communication Engineering Department in U.I.E.T., Panjab University, Chandigarh, India. She has done her B.Sc. and M.Sc. degree in Electronics and Communication Engineering. She got her Ph.D. in the 2013. Her areas of interest are optical communication (wired and wireless), optical biosensors and cognitive neuroscience.

E-mail: preeti_singh@pu.ac.in

Department of Electronics and Communication

Engineering

University Institute of Engineering and Technology

Panjab University

Chandigarh, India

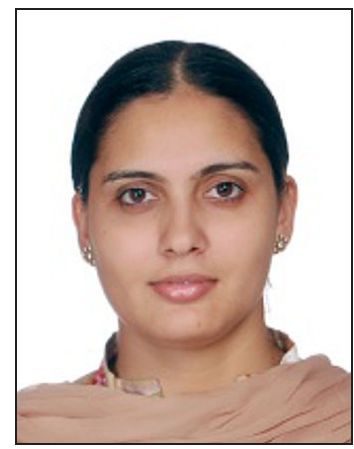

Pardeep Kaur is working as Assistant Professor in Electronics and Communication Engineering Department in U.I.E.T., Panjab University, Chandigarh, India. She has done her B.Sc. and M.Sc. degree in Electronics and Communication Engineering. She is perusing her Ph.D. in wireless sensor networks. Her areas of interest are optical and

wireless communication.

E-mail: pardeep.tur@gmail.com

Department of Electronics and Communication

Engineering

University Institute of Engineering and Technology

Panjab University

Chandigarh, India 